\title{
RECONSTRUCTING THE UNKNOWN LOCAL VOLATILITY FUNCTION*
}

\author{
THOMAS F. COLEMAN $\dagger, \quad$ YUYING LI $\dagger$, AND ARUN VERMA $\dagger$ \\ SEPTEMBER 22, 1998
}

\begin{abstract}
Using market European option prices, a method for computing a smooth local volatility function in a 1-factor continuous diffusion model is proposed. Smoothness is introduced to facilitate accurate approximation of the true local volatility function from a finite set of observation data. It is emphasized that accurately approximating the true local volatility function is crucial in hedging even simple European options, and pricing exotic options. A spline functional approach is used: the local volatility function is represented by a spline whose values at chosen knots are determined by solving a constrained nonlinear optimization problem. The optimization formulation is amenable to various option evaluation methods; a partial differential equation implementation is discussed. Using a synthetic European call option example, we illustrate the capability of the proposed method in reconstructing the unknown local volatility function. Accuracy of pricing and hedging is also illustrated. Moreover, it is demonstrated that, using a different constant implied volatility for an option with different strike/maturity can produce erroneous hedge factors. In addition, real market European call option data on the S\&P 500 stock index is used to compute the local volatility function; stability of the approach is demonstrated.
\end{abstract}

\footnotetext{
* Presented at the first annual conference: Computational and Quantitative Finance '98, New York.

${ }^{\dagger}$ Computer Science Department and Cornell Theory Center, Cornell University, Ithaca, NY 14850. Research partially supported by the Applied Mathematical Sciences Research Program (KC-04-02) of the Office of Energy Research of the U.S. Department of Energy under grant DE-FG02-90ER25013.A000 and NSF through grant DMS-9505155 and ONR through grant N00014-96-1-0050.

This research was conducted using resources of the Cornell Theory Center, which is supported by Cornell University, New York State, the National Center for Research Resources at the National Institutes of Health, and members of the Corporate Partnership Program.
} 
1. Introduction. An option pricing model establishes a relationship between the traded derivatives, the underlying asset and the market variables, e.g., volatility of the underlying asset $[4,24]$. Option pricing models are used in practice to price derivative securities given knowledge of the volatility and other market variables.

The celebrated constant-volatility Black-Scholes model $[4,24]$ is the most often used option pricing model in financial practice. This classical model assumes constant volatility; however, much recent evidence suggests that a constant volatility model is not adequate [27, 26]. Indeed, numerically inverting the Black-Scholes formula on real data sets supports the notion of asymmetry with stock price (volatility skew), as well as dependence on time to expiration (volatility term structure). Collectively this dependence is often referred to as the volatility smile. The challenge is to accurately (and efficiently) model this volatility smile.

In practice, the constant-volatility Black-Scholes model is often applied by simply using different volatility values for options with different strikes and maturities. In this paper, we refer to this approach as the constant implied volatility approach. Although this method works well for pricing European options, it is unsuitable for more complicated exotic options and options with early exercise features. Moreover, as will be illustrated in $\S 4$, this approach can produce incorrect hedge factors even for simple European options.

A few different approaches have been proposed for modeling the volatility smile. One class of methods (Merton [25]) assumes a Poisson jump diffusion process for the underlying asset. Stochastic volatility models (Hull and White [20]) have also been used. Das and Sundaram [10] indicate that neither of these types of models sufficiently explains the implied volatility structure.

Finally, there is the 1-factor continuous diffusion approach: an underlying asset with the initial value $S_{\text {init }}$ is assumed to follow:

$$
\frac{d S_{t}}{S_{t}}=\mu\left(S_{t}, t\right) d t+\sigma^{*}\left(S_{t}, t\right) d W_{t}, \quad t \in[0, \tau], \tau>0
$$

where $W_{t}$ is a standard Brownian motion, $\tau$ is a fixed trading horizon, and $\mu, \sigma^{*}: \Re^{+} \times$ $[0, \tau] \rightarrow \Re$ are deterministic functions. The function $\sigma^{*}(s, t)$ is called the local volatility function. The advantages of the 1 -factor continuous diffusion model, compared to the jump or stochastic model, include that no non-traded source of risk such as the jump or stochastic volatility is introduced [17]. Consequently, the completeness of the model, i.e., the ability to hedge options with the underlying asset, is maintained. Completeness is ultimately important since it allows for arbitrage pricing and hedging [17].

In order to price complex exotic options using a 1-factor diffusion model (1), the volatility function $\sigma^{*}(s, t)$ needs to be approximated. Volatility is the only variable in this 1-factor model which is not directly observable in the market. Similar to the implied volatility in the constant volatility model, one possible idea is to imply this local volatility function from the market option price data. Indeed, it is established $[17,1]$ that the local volatility function can be uniquely determined from the European call options of all strikes and maturities, under the no arbitrage assumption of the observable European call option prices. Unfortunately, the market European option prices are typically limited to a relatively few different strikes and maturities. Therefore the problem of determining the local volatility function can be regarded as a function approximation problem from a finite data set with a nonlinear observation functional. Due to insufficient market option price data, this is a well-known ill-posed problem.

Computational methods have been proposed to solve this ill-posed problem [1, 2, 5, 17, 13, 14, 21, 22, 26]. Most of these methods [1, 5, 17, 13, 14, 21, 26] overcome the ill-posedness of the problem by assuming the existence of a complete spanning set of European call option 
prices, which, in practice, requires use of extrapolation and interpolation of the available market option prices $[26,13,21,5]$. This can be problematic because potentially erroneous non-market information are introduced into the data. Rubinstein proposes to compute the implied probability without any exogenous assumption on the model for the local volatility function [26, 21]. In [1] the local volatility is computed at each discretization nodal point with a PDE approach. The methods [2, 22] use a regularization approach to the ill-posed local volatility approximation problem. The closeness of the local volatility to a prior is used in [2] and smoothness is used in [22].

The local volatility function approximation problem is ill-posed: there are typically an infinite number of solutions to the problem. It is not difficult to find a local volatility function $\sigma(s, t)$ that matches the market option price data. However, for accurately pricing exotic options, we are not merely concerned with matching the market option prices but would like to reconstruct as accurately as possible the the true volatility function $\sigma^{*}(s, t)$ in the diffusion model (1). Accurately approximating the true volatility function is especially important for computing hedge factors, even for simple European call/put options, see $\S 4$.

Smoothness of the function has long been used as a regularization criterion for function approximation with a finite observation data [28, 29, 30]. Splines have known to possess good approximation theoretical properties for a model both when the function is fixed and smooth and when it is a sample function from a stochastic process [30]. However, approximating the local volatility function from a finite set of option prices is more complex, compared to a standard function approximation problem, since the (observation) option price functional is nonlinear. Nevertheless, it is intuitive that smoothness regularization will play a similar role here.

In [22] the lack of sufficient market option price data is overcome by regularizing with smoothness of the local volatility function. The local volatility is computed at each discretization point to match the given option prices with an additional objective of minimizing the change of the derivative $\nabla \sigma(s, t)$. Unfortunately, this approach requires the solution of a very large-scale nonlinear optimization problem: the dimension is equal to the total number of discretization points. In addition, it requires determination of a regularization parameter. Moreover, the computed local volatility function may not be smooth.

In this paper, we propose a spline functional approach: a local volatility function $\sigma(s, t)$ is explicitly represented by a spline with a fixed set of spline knots and end condition. The volatilities at the spline knots uniquely determine a local volatility function. We choose the number of spline knots to be no greater than the number of option prices and they are placed with respect to the given data. The spline is determined by solving a constrained nonlinear optimization problem to match the market option prices as closely as possible. The dimension of the optimization problem is typically small, depending on the number of option prices available. The approximation properties of the spline allow an accurate and smooth approximation of the true local volatility function in a region within which the volatility values are significant for pricing available options.

We start with the motivation for our proposed inverse spline approximation formulation for the local volatility in $\S 2$. Computational issues for solving the proposed optimization problem are discussed in $\S 3$. Numerical examples illustrating the reconstructed local volatility surfaces with the European call option prices are described in $\S 4$. Using a European call option example with the underlying following the known absolute diffusion process, we illustrate the capability of the proposed method for accurately reconstructing the true volatility function. A S\&P 500 European index call option example with the real market data is also used to illustrate the smoothness of the volatility function and the stability of the proposed approach. 
In $\S 5$, concluding remarks are given.

2. Local Volatility Function Approximation with Splines. Assume that the underlying asset follows a continuous 1 -factor diffusion process with the initial value $S_{\text {init }}$ :

$$
\frac{d S_{t}}{S_{t}}=\mu\left(S_{t}, t\right) d t+\sigma^{*}\left(S_{t}, t\right) d W_{t}, \quad t \in[0, \tau],
$$

for some fixed time horizon $[0, \tau], W_{t}$ is a Brownian motion, and $\mu(s, t), \sigma^{*}(s, t): \Re^{+} \times$ $[0, \tau] \rightarrow \Re$ are deterministic functions sufficiently well behaved to guarantee that (1) has a unique solution [23]. Note that in this notation $\sigma^{*}(s, t)$ can be negative as well as positive. (The conventional notion of positive volatility corresponds to $\sqrt{\sigma^{*}(s, t)^{2}}$ in our notation.) For simplicity, we assume that the instantaneous interest rate is a constant $r>0$ and the dividend rate is a constant $q>0$ (A general stochastic interest derivative pricing is described in [19]). Given $S_{\text {init }}, r$ and $q$, and under the no arbitrage assumption [24], an option with the volatility $\sigma(s, t)$, strike price $K$, and maturity $T$ has a unique price $v(\sigma(s, t), K, T)$.

Assume that we are given $m$ market option (bid,ask)-pairs, $\left\{\left(\operatorname{bid}_{j}, \text { ask }_{j}\right)\right\}_{j=1}^{m}$, corresponding to strike prices/expiration times $\left\{\left(K_{j}, T_{j}\right)\right\}_{j=1}^{m}$. Let

$$
v_{j}(\sigma(s, t)) \stackrel{\text { def }}{=} v\left(\sigma(s, t), K_{j}, T_{j}\right), \quad j=1, \cdots, m .
$$

We want to approximate, as accurately as possible, the true local volatility function $\sigma^{*}(s, t)$ : $\Re^{+} \times[0, \tau] \rightarrow \Re$ from the requirement that

$$
\operatorname{bid}_{j} \leq v_{j}(\sigma(s, t)) \leq \operatorname{ask}_{j}, \quad j=1, \cdots, m .
$$

Since the observation data $\left\{\left(\operatorname{bid}_{j}, \operatorname{ask}_{j}, K_{j}, T_{j}\right)\right\}_{j=1}^{m}$ is finite and the restriction is on the option values $\left\{v_{j}(\sigma(s, t))\right\}_{j=1}^{m}$, problem (2) can be considered an inverse function approximation problem from a finite observation data. Let $\mathcal{H}$ denote the space of measurable functions in the region $[0,+\infty) \times[0, \tau]$. The inverse function approximation problem (2) can be written as an optimization problem:

$$
\left.\left.\min _{\sigma(s, t) \in \mathcal{H}} \sum_{j=1}^{m}\left[\operatorname{bid}_{j}-v_{j}(\sigma(s, t))\right)\right]^{+}+\sum_{j=1}^{m}\left[v_{j}(\sigma(s, t))-\operatorname{ask}_{j}\right)\right]^{+},
$$

where $x^{+} \stackrel{\text { def }}{=} \max (x, 0)$. This is a nonlinear piecewise differentiable optimization problem: to overcome nondifferentiability in (3), one can alternatively solve a variational least squares problem:

$$
\min _{\sigma(s, t) \in \mathcal{H}} \sum_{j=1}^{m}\left(v_{j}(\sigma(s, t))-\bar{v}_{j}\right)^{2}
$$

where $\bar{v}_{j} \stackrel{\text { def }}{=} \frac{\text { bid }_{j}+\mathrm{ask}_{j}}{2}$. Since the observation data is finite, problems $(2,3,4)$ are severely underdetermined: there are typically infinite number of solutions. It is easy to find a function $\sigma(s, t)$ that matches the market option price data $[2,5,17,13,14,21,22,26]$.

The local volatility reconstruction problem $(2,3,4)$ is a complicated nonstandard function approximation problem. The option price functional $v(\sigma(s, t), K, T)$ is nonlinear in the local volatility function $\sigma(s, t)$. It is a nonlinear inverse function approximation problem.

In most of the proposed methods $[1,2,5,17,13,14,21,26]$ matching the market option price data has been emphasized; it is often the only objective. However, a function $\sigma(s, t)$ 
which matches the finite set of market option prices can be very different from the true local volatility $\sigma^{*}(s, t)$, see $\S 4$ for an example. Moreover, the price $\bar{v}_{j}$ generally has error (for example when a bid-ask spread exists). In addition, the option value $v_{j}(\sigma(s, t))$ can only be computed numerically using a tree method or a PDE approach (there is no closed form solution for a general 1-factor model (1)). Hence, it may not be desirable to insist that $v_{j}(\sigma(s, t))$ match exactly the observed market price $\bar{v}_{j}$ for $j=1, \cdots, m$. For pricing and hedging of exotic options, it is more important to compute a local volatility function $\sigma(s, t)$ which is close to the true local volatility function $\sigma^{*}(s, t)$. In other words, in addition to calibrating the market option price data sufficiently accurately, we would like to reconstruct the true local volatility function $\sigma^{*}(s, t)$ of the diffusion model (1) as accurately as possible.

Smoothness has long been used $[28,29,30]$ as a regularization condition for a function approximation problem with a limited observation data. In addition, smoothness of the local volatility function can be important in computational option valuation schemes. Convergence of a PDE finite difference method, for example, depends on the smoothness of the function $\sigma(s, t)$.

In [22] it is proposed to use smoothness as a regularization condition to approximate the local volatility function. The regularized optimization problem

$$
\left.\min _{\sigma(s, t) \in \mathcal{H}} \sum_{j=1}^{m}\left(v_{j}(\sigma(s, t))\right)-\bar{v}_{j}\right)^{2}+\lambda\|\nabla \sigma(s, t)\|_{2}
$$

is used in [22] where $\lambda$ is a positive constant and $\|\cdot\|_{2}$ denotes the $L^{2}$ norm. The change of the first order derivative is minimized depending on the regularization parameter $\lambda$ for which determining a suitable value may not be easy. In addition, computational implementation of this method requires solving a large-scale discretized optimization problem: for a PDE implementation, the dimension is $N M$ where $N$ is the number of discretization points in $s$ and $M$ is the number of discretization points in $t$. A simple gradient descent algorithm is used in [22]. Since the optimization problem is (5) highly nonlinear, with such a method, the computed solution is typically inaccurate. To use a more sophisticated optimization algorithm, the Jacobian matrix of the vector function $\left(v_{1}, \cdots, v_{m}\right)$ needs to be evaluated but this becomes extremely costly due to the large dimension of the discretized problem.

Splines have long been used in approximating smooth curves and surfaces (see, e.g., [16]). They have also been used as a tool for regularizing ill-posedness of function approximations from finite observation data [30]. In a typical 1-dimensional spline interpolation setting, assuming values $f_{i}, i=1, \cdots, m$, of the dependent variable $f(x)$ corresponding to values $x_{i}, i=1, \cdots, m$, are given, a spline is chosen to fit the data $\left(f_{i}, x_{i}\right), i=1, \cdots, m$. Given the number of knots $p$ and their locations, the freedom of the spline is the coefficient of each spline segment. The cubic spline has long been used by craftsman and engineers as the mechanic spline. It is the smoothest twice continuously differentiable function that matches the observations: the minimizer of

$$
\min _{f(x) \in \mathcal{S}} \int_{a}^{b}\left(f^{\prime \prime}(x)\right)^{2} d x, \quad \text { subject to } f\left(x_{i}\right)=f_{i}, \quad i=1, \cdots, m,
$$

is a natural cubic spline, where $\mathcal{S}$ is the Sobolev space of functions whose first order derivatives are continuously differentiable and the second order derivatives are square integrable (assuming $m \geq 2$ ). For mechanical splines, this corresponds to minimize the elastic strain energy. For 2-dimensional surface fitting, the bicubic spline defined on a regular grid is twice continuously differentiable $[3,16]$. The bicubic spline has a similar variational minimization 
property. Advantages of spline interpolation include its fast convergence on many types of meshes, computational efficiency, and insensitivity to roundoff errors [3].

Approximating the local volatility function by a spline is particularly reasonable if the local volatility function is smooth. Is this a reasonable expectation for the local volatility function? Assume that the underlying follows the 1-factor diffusion process (1). Let there be given observable arbitrage-free market European call prices $v(K, T)$ for all strikes $K \in$ $[0, \infty)$ and all maturities $T \in(0, \tau]$. From Proposition 1 in [1], the local volatility function $\sigma^{*}(s, t)$ of the diffusion process (1) that is consistent with the market is given uniquely by

$$
\left(\sigma^{*}(K, T)\right)^{2}=2 \frac{\frac{\partial v}{\partial T}+q v(K, T)+K(r-q) \frac{\partial v}{\partial K}}{K^{2} \frac{\partial^{2} v}{\partial K^{2}}} .
$$

This formula suggests that, assuming $v(K, T)$ is sufficiently smooth (note that $\frac{\partial^{2} v}{\partial K^{2}}$ and $\frac{\partial v}{\partial T}$ already exist), $\left(\sigma^{*}(K, T)\right)^{2}$ is sufficiently smooth in the region $(0, \infty) \times(0, \tau]$ as well.

In this paper, we use a 2-dimensional spline functional to directly approximate a local volatility function ${ }^{1}$. Let the number of spline knots $p \leq m$. We choose a set of fixed spline knots $\left\{\left(\bar{s}_{j}, \bar{t}_{j}\right)\right\}_{j=1}^{p}$ in the region $[0, \infty) \times[0, \tau]$. Given $\left\{\left(\bar{s}_{i}, \bar{t}_{i}\right)\right\}_{i=1}^{p}$ spline knots with corresponding local volatility values $\bar{\sigma}_{i} \stackrel{\text { def }}{=} \sigma\left(\bar{s}_{i}, \bar{t}_{i}\right)$, an interpolating cubic spline $c(s, t)$ with a fixed end condition (in our computation the natural spline end condition is used) is uniquely defined by setting $c\left(\bar{s}_{i}, \bar{t}_{i}\right)=\bar{\sigma}_{i}, i=1, \cdots, p$. We then determine the local volatility values $\bar{\sigma}_{i}$ (hence the spline) by calibrating the market observable option prices. The freedom in this problem is represented by the volatility values $\left\{\bar{\sigma}_{i}\right\}$ at the given knots $\left\{\left(\bar{s}_{i}, \bar{t}_{i}\right)\right\}$. If $\bar{\sigma}$ is a $p$-vector, $\bar{\sigma}=\left(\bar{\sigma}_{1}, \cdots, \bar{\sigma}_{p}\right)^{T}$, then we denote the corresponding interpolating spline with the specified end condition as $c(s, t ; \bar{\sigma})$.

Let

$$
v_{j}(c(s, t ; \bar{\sigma})) \stackrel{\text { def }}{=} v\left(c(s, t ; \bar{\sigma}), K_{j}, T_{j}\right), j=1, \cdots, m .
$$

To allow the possibility of incorporating additional a priori information, $l$ and $u$ are lower and upper bounds that can be imposed on the local volatilities at the knots. Thus, we define the inverse spline local volatility approximation problem: Given $p$ spline knots, $\left(\bar{s}_{1}, \bar{t}_{1}\right) \cdots,\left(\bar{s}_{p}, \bar{t}_{p}\right)$, solve for the $p$-vector $\bar{\sigma}$

$$
\min _{\bar{\sigma} \in \Re^{p}} f(\bar{\sigma}) \stackrel{\text { def }}{=} \frac{1}{2} \sum_{j=1}^{m} w_{j}\left[v_{j}(c(s, t ; \bar{\sigma}))-\bar{v}_{j}\right]^{2}
$$

subject to $\quad l \leq \bar{\sigma} \leq u$,

where positive constants $\left\{w_{j}\right\}_{j=1}^{m}$ are weights, allowing account to be taken of different accuracies of $\bar{v}_{j}$ or computed $v_{j}$. The determination of an approximation in the $l_{1}$ or $l_{\infty}$ norm instead may be a valuable alternative although the problem becomes even more difficult to solve computationally. Note also that the formulation (7) is quite general: European call/put or even more complicated option prices can be used to compute the spline volatility approximation to the local volatility function $\sigma^{*}(s, t)$.

The inverse spline local volatility problem (7) is a minimization problem with respect to the local volatility $\bar{\sigma}$ at the spline knots. The computed volatility function has some dependence on the number of knots $p$ and the location of the knots $\left\{\left(\bar{s}_{i}, \bar{t}_{i}\right)\right\}_{i=1}^{p}$. The choice of the

\footnotetext{
${ }^{1}$ If it is known that $\sigma(s, t)$ is a function of $s$ or $t$ only, then one can use 1-dimensional spline.
} 
number of knots and their placement in spline approximation is generally a complicated issue $[16,30]$. The situation here is not typical for spline approximation due to the fact that the dependent option price function is not the function to be approximated. Rather, it depends on the values of the unknown volatility function in the region $R^{+} \times[0, \tau]$. Moreover, the dependence on the unknown volatility values is not uniform in the region $R^{+} \times[0, \tau]$. The option premium depends little on the volatility values with small $t$ and $s$ far from $S_{\text {init }}$. It is convenient to view this as follows [1]: there exists a region centered around $S_{\text {init }}$ within which the volatility values are significant in pricing and hedging: we denote this region as $\mathcal{D}_{j}$ for the option $v_{j}$, see Fig. 1 for illustration of its typical shape. We can at most expect to approximate well the local volatilities in the region $\mathcal{D} \stackrel{\text { def }}{=} \cup_{j=1}^{m} \mathcal{D}_{j}$ from the market option data. In our experiments, we often choose the number of knots equal to the number of observations. In order to construct and evaluate a spline efficiently, the spline knots can be placed in a rectangular mesh covering the region $\mathcal{D}$ and bicubic spline interpolation [3] can be used.

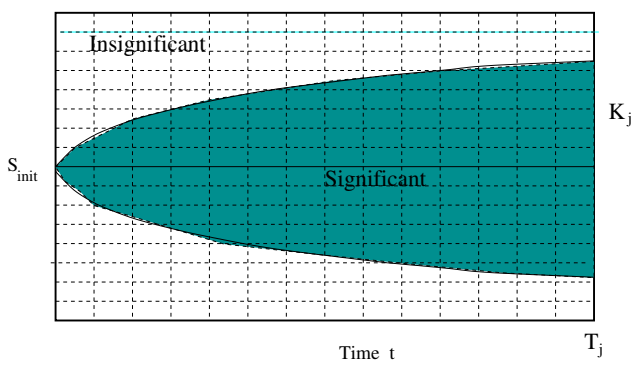

FIG. 1. The Local Volatility in the Shaded Region $\mathcal{D}_{j}$ Is Significant in Pricing and Hedging

If the number of spline knots are chosen to be no more than the number of observation data points, the degrees of freedom, compared to that of a (discretized) formulation of (3), is significantly decreased (several orders of magnitude). In addition to gaining smoothness of the local volatility function, formulation (7) significantly decreases the computational cost compared to that of the (discretized) formulations $(3,5)$ due to reduction of the dimension of the optimization problem.

It is not appropriate to choose $p$ much larger than $m$ since (7) may become underdetermined. If one decides to use more spline knots, additional regularization, e.g.,

$$
\min _{\bar{\sigma} \in \Re^{p}} f(\bar{\sigma}) \stackrel{\text { def }}{=} \frac{1}{2} \sum_{j=1}^{m} w_{j}\left[v_{j}(c(s, t ; \bar{\sigma}))-\bar{v}_{j}\right]^{2}+\lambda \eta(c(s, t ; \bar{\sigma}))
$$

subject to $\quad l \leq \bar{\sigma} \leq u$,

is more appropriate: here $\lambda>0$ is a regularization parameter and $\eta(\sigma(s, t ; \sigma))$ is a smoothing norm for the tensor product splines [15].

In this paper, we focus on the formulation (7) and assume $p$ is not greater than $m$. In order to solve the inverse local volatility problem (7), an optimization method will be needed to evaluate the values of options $v_{j}(c(s, t ; \bar{\sigma}))$ for any spline $c(s, t ; \bar{\sigma})$; the derivatives may also be computed. We discuss this next.

3. The Computational Procedure. Our proposal is to approximate the local volatility surface, $\sigma^{*}(s, t)$, with a cubic spline $c(s, t ; \bar{\sigma})$ by solving $(7)$ for the vector $\bar{\sigma}=\left(\bar{\sigma}_{1}, \cdots, \bar{\sigma}_{p}\right)^{T}$. Problem (7), when $p \leq m$, is well-defined once the $p$ knots $\left(\bar{s}_{1}, \bar{t}_{1}\right), \cdots,\left(\bar{s}_{p}, \bar{t}_{p}\right)$ have been chosen appropriately. To express (7) more succinctly, define a vector-valued function $F$ : 
$\Re^{p} \rightarrow \Re^{m}$ where component $j$ of $F$ is given by $w_{j}^{\frac{1}{2}}\left[v_{j}(c(s, t ; \bar{\sigma}))-\bar{v}_{j}\right]$, for $j=1, \cdots, m$. Therefore (7) can be rewritten:

$$
\begin{array}{ll} 
& \min _{\bar{\sigma} \in \Re^{p}} f(\bar{\sigma}) \stackrel{\text { def }}{=} \frac{1}{2}\|F(\bar{\sigma})\|_{2}^{2} \\
\text { subject to } & l \leq \bar{\sigma} \leq u .
\end{array}
$$

Problem (9) is a box-constrained nonlinear least-squares problem in $\bar{\sigma}$; there are a variety of optimization methods available to enable its solution. In our implementation we use a trust region/interior method based on [7,6], in which a sequence of strictly interior points are generated: $\left\{\bar{\sigma}^{(k)}\right\} \in \operatorname{int}\{\mathcal{F}\}$, where $\mathcal{F}=\left\{\bar{\sigma} \in \Re^{p}: l \leq \bar{\sigma} \leq u\right\}$. Moreover, the sequence corresponds to a monotonically decreasing sequence of function values, i.e., $f^{(k+1)}<f^{(k)}, k=1, \cdots, \infty$, where $f^{(k)}=f\left(\bar{\sigma}^{(k)}\right)$. Under mild assumptions this approach guarantees convergence, i.e., $\bar{\sigma}^{(k)} \rightarrow \bar{\sigma}^{*}$, where $\bar{\sigma}^{*}$ is a local minimizer for problem (9).

The Jacobian of $F$ with respect to $\bar{\sigma}$ is required: $J(\bar{\sigma}) \stackrel{\text { def }}{=} \nabla F(\bar{\sigma})$. Note that $J$ is an $m \times p$ matrix. In the square case when $p=m$, it is possible to use a standard secant update to approximate $J$, e.g., [12], which can significantly reduce the cost. Under reasonable assumptions a superlinear rate of convergence can be achieved. We note that there are optimization approaches that do not require the calculation (or approximation) of the Jacobian matrix $J$; however, they typically converge very slowly - we have not investigated those methods in this work.

In this paper we explore two possibilities in the framework of our optimization approach:

1. Use of automatic differentiation [8] and/or finite-differencing to compute $J^{(k)} \stackrel{\text { def }}{=}$ $J\left(\bar{\sigma}^{(k)}\right)$;

2. Use of a secant update to approximate $J^{(k)}$ when $p=m$.

3.1. The Problem Structure. The evaluation of $f(\bar{\sigma})$ requires the evaluation of each component of $F$, i.e., $w_{j}^{\frac{1}{2}}\left[v_{j}(c(s, t) ; \bar{\sigma})-\bar{v}_{j}\right]$, for $j=1, \cdots, m$. These are generalized BlackScholes computations. There are several ways to approach this - we choose, as an example, to use a standard PDE-discretization technique.

Given $S_{\text {init }}, r, q$, and $\sigma(s, t)$, let $V(s, t)$ denote the option value of an underlying asset with strike price $K$ and expiry date $T$ at $(s, t), t \in[0, T]$. Under the no arbitrage assumption, the option value satisfies the following generalized Black-Scholes equation [24]

$$
\frac{\partial V}{\partial t}+(r-q) s \frac{\partial V}{\partial s}+\frac{1}{2} \sigma(s, t)^{2} s^{2} \frac{\partial^{2} V}{\partial s^{2}}=r V .
$$

The boundary conditions for the European call option are :

$$
\begin{array}{r}
\lim _{s \rightarrow+\infty} \frac{\partial V(s, t)}{\partial s}=e^{-q(T-t)}, \quad t \in[0, T], \\
V(0, t)=0, \quad t \in[0, T], \\
V(s, T)=\max (s-K, 0) .
\end{array}
$$

We use a Crank-Nicholson finite difference solution strategy for solving (10), based on discretization on a uniform grid. Given a 2-dimensional grid the numerical solution of (10) is standard and discussed in several texts. Zvan et al [31] have a good discussion of complexity issues. It is possible to increase efficiency by employing a number of computing techniques such as vectorization and pipelining - description of these implementation aspects goes beyond the scope of this paper. 
3.2. Computing the Jacobian and the gradient. The Jacobian matrix $J(\bar{\sigma})$ satisfies

$$
J(\bar{\sigma})=\frac{\partial v}{\partial c} \times \frac{\partial c}{\partial \bar{\sigma}}
$$

where $\frac{\partial v}{\partial c}$ is an $m$-by- $M N$ matrix, $\frac{\partial c}{\partial \bar{\sigma}}$ is an $M N$-by- $p$ matrix.

It is useful to note that matrix $C \stackrel{\text { def }}{=} \frac{\partial c}{\partial \bar{\sigma}}$ is constant and therefore needs to be computed just once for the entire problem (given a fixed discretization and spline knot placement). The product $\frac{\partial v}{\partial c} \times C$ can be computed directly using automatic differentiation (forward mode) or approximately using finite differences (differencing $v$ along the columns of $C$ ). In either case the work involved is $O(p \cdot \omega(F))$ where $\omega(F)$ is the work (flops) required to evaluate $F$. (In the finite-differencing case this is a tight bound whereas this bound can be undercut considerably if automatic differentiation is used [18].)

The gradient of $f$, with respect to $\bar{\sigma}$, is simply $J^{T} F$. Therefore if the function $F$ and its Jacobian $J$ have been computed as described above the gradient is given by a matrix-vector multiplication.

If the secant method is used in the square case, i.e., $p=m$, then the gradient is approximated by $A \times F$ where $A$ is the secant approximation to the Jacobian. The Jacobian is not computed (except, possibly, for secant method restarts) with this approach.

4. Computational Examples. We now describe some computational experience with our proposed method for reconstructing the local volatility function $\sigma^{*}(s, t)$ from limited observation data. We illustrate how European call options can be used to approximate the true local volatility function.

We have implemented the proposed method in Matlab using a trust region optimization algorithm with a PDE approach for function and Jacobian evaluation. Without precise knowledge of accuracy of the market data, the weights in the inverse spline local volatility approximation problem (7) are simply set to unity: $w_{j} \stackrel{\text { def }}{=} 1, j=1, \cdots, m$. The generalized Black-Scholes PDE (10) is solved with a Crank-Nicolson finite difference method. Given any $\bar{\sigma}$, the bicubic spline $c(s, t ; \bar{\sigma})$ with the variational end condition (the second order derivative at the end is zero) is computed and evaluated using the functions in the Matlab spline toolbox [11]. We use a simple discretization scheme: a uniformly spaced mesh with $N \times M$ grid points in the region $\left[0,2 S_{\text {init }}\right] \times[0, \tau]$ where $\tau$ is the maximum maturity in the market option data:

$$
\begin{aligned}
s_{i}=i \frac{2 S_{\text {init }}}{N-1}, & i=0, \cdots, N-1, \\
t_{j}=j \frac{\tau}{M-1}, & j=0, \cdots, M-1 .
\end{aligned}
$$

For simplicity, we have chosen the spline knots to be on a uniform rectangular mesh covering the region $\mathcal{D}$ in which the volatility values are significant in pricing the market options. Given a European option, we do not have an explicit knowledge of the region $\mathcal{D}$. In our experiments, we have used $\left[\gamma_{1} S_{\text {init }}, \gamma_{2} S_{\text {init }}\right] \times[0, \tau]$ as an estimate of $\mathcal{D}$ with $\gamma_{1} \in[.6, .8]$ and $\gamma_{2} \in[1.4,1.6]$ depending on the magnitude of $S_{\text {init }}$. The number of spline knots $p$ typically equals the number of observation $m$. In the event that the market option prices are calibrated to high precision, we have experimented with $p<m$.

4.1. Reconstructing Local Volatility, Pricing and Hedging. In order to demonstrate the effectiveness of the proposed method in reconstructing the true local volatility surface and its accuracy in pricing and hedging, we consider a synthetic European call option example 
used in [22]. In this example, the underlying is assumed to follow an absolute diffusion process:

$$
\frac{d S_{t}}{S_{t}}=\mu\left(S_{t}, t\right) d t+\sigma^{*}\left(S_{t}, t\right) d W_{t}
$$

where the local volatility function $\sigma^{*}(s, t)$ is a function of the underlying only,

$$
\sigma^{*}(s, t)=\frac{\alpha}{s},
$$

with $\alpha=15$, and $W_{t}$ representing a standard Brownian motion. We use the same parameter setting as in [1]: Let the initial stock index be $S_{\text {init }}=100$, the risk free interest rate $r=0.05$ and the dividend rate $q=0.02$.

We consider, as market option data, 22 European call options on the underlying following the absolute diffusion process (12). Eleven options have half year maturity with strike prices $[90: 2: 110]$ and another eleven options have one year maturity with the same strikes. Thus the option strike and maturity vectors are given below

$$
\begin{aligned}
& K=[90 ; 92 ; \cdots ; 110 ; 90 ; 92 ; \cdots ; 110] \in \Re^{22}, \\
& T=[0.5 ; 0.5 ; \cdots ; 0.5 ; 1 ; 1 ; \cdots ; 1] \in \Re^{22} .
\end{aligned}
$$

For the absolute diffusion process (12), the analytic formula for pricing European options exists [9] and we set the market European option call price $\bar{v}_{j}$ equal to this analytic value. The discretization parameters in (11) are set as $M=101$ and $N=51$.
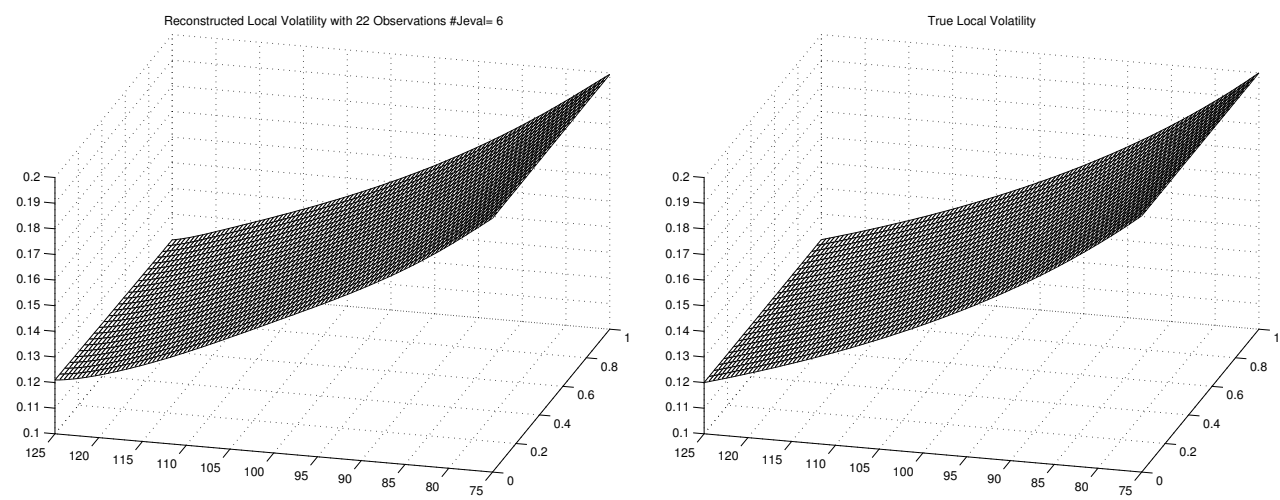

FIG. 2. The Reconstructed and True Local Volatility

For this example the lower and upper bounds for the local volatility at the knot $\bar{\sigma}_{i}$ are $l_{i}=-1$ and $u_{i}=1$ respectively (though no variable is at the bound at the computed solution in this case). First, we let $p=m$ and place the spline knots on the grid $[0: 20: 200] \times[0,1]$. The initial volatility values at knots are specified as $\bar{\sigma}_{i}^{(0)}=0.15, i=1, \cdots, 22$. The resulting optimization problem is relatively easy to solve. The optimization method requires 7 iterations (6 Jacobian evaluations) and the computed optimal objective function value $f\left(\bar{\sigma}^{*}\right)$ is $10^{-6}$.

Fig. 2 demonstrates the accuracy of the local volatility reconstruction: the reconstructed spline surface $c\left(s, t ; \bar{\sigma}^{*}\right)$ very accurately approximates the the actual volatility surface $\sigma^{*}(s, t)$ in the neighborhood of the region $[75,125] \times[0,1]$. To better observe accuracy of reconstruction, the three plots on the left in Fig. 3 display the local volatility curves for $t=0,0.58$ and 
$t=1$ respectively. Since the calibration error is very small and the reconstructed volatility surface is nearly linear, we experimented with choosing the number of spline knots less than $m$. The three plots on the right of Fig. 3 display the local volatility curves reconstructed with eight spline knots placed on the mesh $\left[.4 S_{\text {init }}: .4 S_{\text {init }}: 1.6 S_{\text {init }}\right] \times[0,1]$. We observe that the local volatility reconstruction remains excellent, with a slightly larger deviation when $t$ is small and $s$ is far from $S_{\text {init }}$.
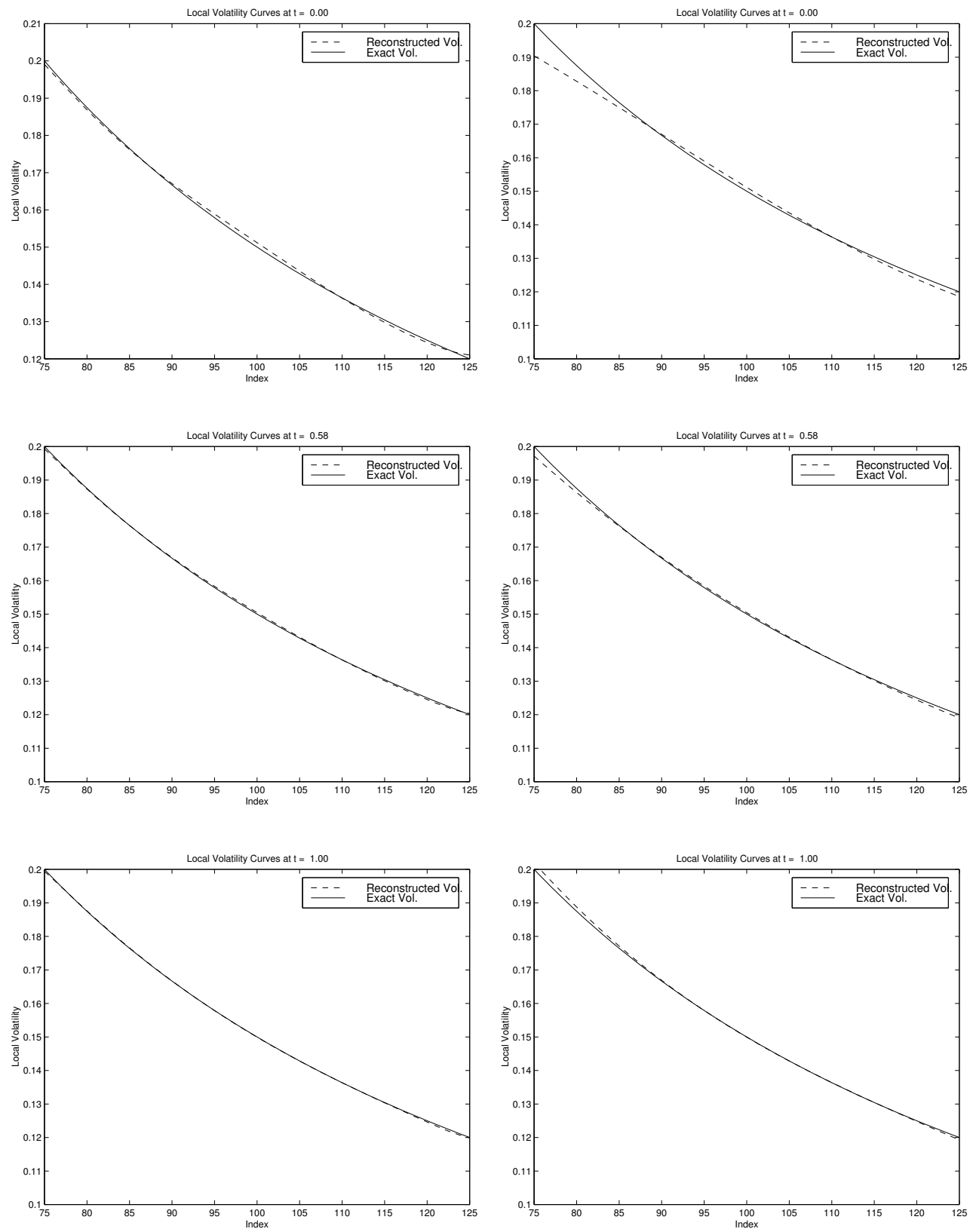

FIG. 3. Left: Knots $=[0: 20: 200] \times[0,1]$, Right: Knots $=\left[.4 S_{\text {init }}: .4 S_{\text {init }}: 1.6 S_{\text {init }}\right] \times[0,1]$

To illustrate the accuracy of pricing using the reconstructed local volatility $c\left(s, t ; \bar{\sigma}^{*}\right)$ rather than the true local volatility $\sigma^{*}(s, t)$, we compare prices and hedge factors of a number of European call options using both the true and reconstructed volatility surfaces. The hedge factors vega (sensitivity to the change in volatility), delta (sensitivity to the change under- 


\begin{tabular}{|l|r|r|}
\hline & max relative error & average relative error \\
\hline Price & $7.8 e^{-3}$ & $2.1 e^{-3}$ \\
\hline Vega & $9.8 e^{-3}$ & $6.1 e^{-3}$ \\
\hline Delta & $4.8 e^{-2}$ & $1.3 e^{-2}$ \\
\hline Gamma & $9.5 e^{-2}$ & $5.9 e^{-2}$ \\
\hline Rho & $4.5 e^{-3}$ & $2.0 e^{-3}$ \\
\hline Theta & $6.9 e^{-3}$ & $2.2 e^{-3}$ \\
\hline
\end{tabular}

TABLE 1

Accuracy of Pricing and Hedging

lying), gamma (sensitivity of delta to change in the underlying), rho (sensitivity to change in the interest rate) and theta (sensitivity to change in the maturity) are computed using a finite difference approximation. A constant shift in both volatility surfaces is used to calculate the vega hedge factor. For European call options with strikes and maturities over the grid $[85: 5: 110] \times[.4: .1: .7]$, the results are shown in Table 1 . They indicate that fairly accurate prices as well as hedge factors are obtained using the reconstructed volatility surface $c\left(s, t ; \bar{\sigma}^{*}\right)$. Note that the PDE option evaluation with the chosen discretization can generate errors of at least these magnitudes.

We emphasize that the formulation (7) is appropriate when the number of spline knots $p$ is not greater than the number of observations $m$. If $p$ is much larger than $m$, then formulation (7) can become severely underdetermined. To illustrate the potential pitfalls of allowing too much freedom in approximating $\sigma^{*}(s, t)$, we simulate the more realistic market situation when there is a bid-ask spread in the given option prices by setting

$$
\bar{v}_{j}=\text { exact price of option } j+.02 \text { rand }
$$

where rand is a Matlab generated random number. We compare the local volatility reconstructions using the spline knots on the rectangular meshes $\left[.4 S_{\text {init }}: .4 S_{\text {init }}: 1.6 S_{\text {init }}\right] \times[0,1]$ $(p=8)$ and $[0: 2: 200] \times[0,1](p=202<M N)$. The plots on the left in Fig. 4 illustrate the reconstructed local volatility curves using the rectangular mesh $\left[.4 S_{\text {init }}: .4 S_{\text {init }}: 1.6 S_{\text {init }}\right] \times[0,1]$ for knots. The plots on the right in Fig. 4 illustrate the reconstructed local volatility curves using the rectangular mesh $\left[0: 2: 2 S_{\text {init }}\right] \times[0,1]$ for knots. Although the available option prices are matched with very high accuracy (error about $10^{-6}$ ) using $p=202$, the computed local volatility surface does not resemble the true local volatility surface $\sigma^{*}(s, t)$. Using eight knots on the rectangular mesh $\left[.4 S_{\text {init }}: .4 S_{\text {init }}: 1.6 S_{\text {init }}\right] \times[0,1]$, on the other hand, yields a much more accurate volatility surface, even though the calibration error of the available options is larger (about $10^{-4}$ ).

Next we illustrate that a constant implied volatility approach can produce incorrect hedge factors even though the option prices may be computed accurately. We use the same absolute diffusion model (12) but with greater volatility: the constant $\alpha=75$ is used instead of $\alpha=15$. The same initial underlying $S_{\text {init }}=100$ and the risk free interest rate $r=0.05$ are used but the dividend rate $q$ is set to zero. We consider European call options with strikes and maturities at the grid $[80: 4: 120] \times[.25, .5,1]$. The spline knots are at the grid $\left[0: 20: 2 S_{\text {init }}\right] \times[.25, .5,1]$. Fig. 5 displays the price and hedge factors of options with maturity .25 year using the true volatility, reconstructed volatility, and constant implied volatility. From these plots, we see that the price and all the hedge factors computed using the reconstructed volatility function are fairly accurate approximation to the true values. Using the constant implied volatility method, however, large errors exist in hedge factors (mostly noticeably in theta, delta, gamma and vega). 

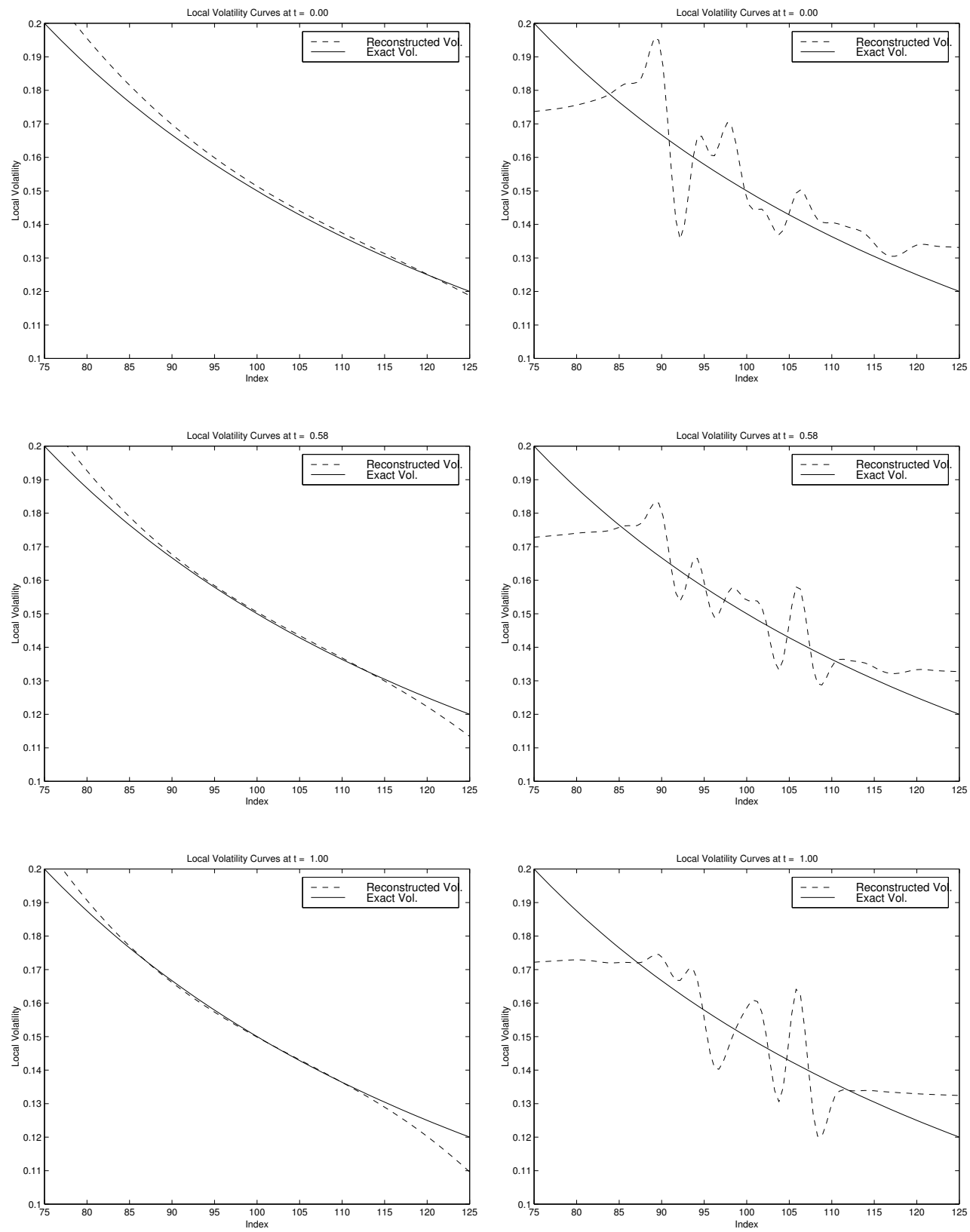

FIG. 4. Left: Knots $=\left[.4 S_{\text {init }}: .4 S_{\text {init }}: 1.6 S_{\text {init }}\right] \times[0,1]$, Right: Knots $=[0: 2: 200] \times[0,1]$ 


\begin{tabular}{|c|c|c|c|c|c|c|c|c|c|c|}
\hline Maturity (in years) & \multicolumn{9}{|c|}{ Strike (\% of spot) } \\
\hline & $85 \%$ & $90 \%$ & $95 \%$ & $100 \%$ & $105 \%$ & $110 \%$ & $115 \%$ & $120 \%$ & $130 \%$ & $140 \%$ \\
\hline .175 & .190 & .168 & .133 & .113 & .102 & .097 & .120 & .142 & .169 & .200 \\
\hline .425 & .177 & .155 & .138 & .125 & .109 & .103 & .100 & .114 & .130 & .150 \\
\hline .695 & .172 & .157 & .144 & .133 & .118 & .104 & .100 & .101 & .108 & .124 \\
\hline .94 & .171 & .159 & .149 & .137 & .127 & .113 & .106 & .103 & .100 & .110 \\
\hline 1 & .171 & .159 & .150 & .138 & .128 & .115 & .107 & .103 & .099 & .108 \\
\hline 1.5 & .169 & .160 & .151 & .142 & .133 & .124 & .119 & .113 & .107 & .102 \\
\hline 2 & .169 & .161 & .153 & .145 & .137 & .130 & .126 & .119 & .115 & .111 \\
\hline 3 & .168 & .161 & .155 & .149 & .143 & .137 & .133 & .128 & .124 & .123 \\
\hline 4 & .168 & .162 & .157 & .152 & .148 & .143 & .139 & .135 & .130 & .128 \\
\hline 5 & .168 & .164 & .159 & .154 & .151 & .148 & .144 & .140 & .136 & .132 \\
\hline
\end{tabular}

TABLE 2

Implied Volatilities for $S \& P$ Index Call Options

In addition to choosing the number of spline knots $p$, the placement of the knots requires some care as well. The spline knots should be placed to cover the region $\mathcal{D}$ within which the values of the local volatility are significant in the option values. We have used the uniform spacing in the interval $\left[0,2 S_{\text {init }}\right]$ and $\left[.4 S_{\text {init }}, 1.6 S_{\text {init }}\right]$ in this synthetic example but an alternative is to place them nonuniformly with a more refined placement around $s=S_{\text {init }}$. Moreover, one need to avoid placing spline knots too closely together since this can lead to ill conditioning of the Jacobian matrix $\nabla F$.

4.2. A S\&P 500 Example Illustrating Smoothness and Stability. We consider now a more realistic example of approximating the local volatility function $\sigma^{*}(s, t)$ from the European S\&P 500 index call options. We use the same European call option data of October 1995 given in [1]. The market option price data (in the implied Black-Scholes constant volatility) is given in Table 2. Similar to [1], we use only the options with no more than two years maturity in our computation. The initial index, interest rate and dividend rate are set as in [1],

$$
S_{\text {init }}=\$ 590, \quad r=0.06, \quad \text { and } q=0.0262 .
$$

The discretization parameters in (11) are set

$$
N=101, \quad \text { and } \quad M=101 .
$$

In order to solve the proposed inverse spline volatility problem (7), we compute the market European call option prices with given strikes and maturities using the constant volatility Black-Scholes formula with the corresponding implied volatility. The Matlab function blsprice is used.

For this example the number of spline knots $p$ equals the number of observations $m$ and the spline knots are placed on a rectangular mesh $\left[.8 S_{\text {init }}: .066 S_{\text {init }}: 1.4 S_{\text {init }}\right] \times[0: .33: 2]$. Using all the call option prices with maturity $T \leq 2$ in Table 2 , the reconstructed local volatility surface is given in Fig. 6 . This optimization problem seems to be more nonlinear and difficult to solve. After 28 iterations, the average error of $v_{j}(c(s, t ; \bar{\sigma}))-\bar{v}_{j}$ using the reconstructed local volatility is 0.0076 . The average error using the constant implied volatility via the PDE implementation with this discretization, compared to the Black-Scholes analytic formula, is 0.0510 .

The reconstructed local volatility surfaces can be slightly different if different spline knots are chosen. In order to show that volatility surface reconstruction, pricing and hedging are relatively robust, we consider the second spline knots placement using the rectangular mesh 


\begin{tabular}{|l|r|r|}
\hline & max rel. diff. & avg. rel. diff. \\
\hline Price & $6.8 e^{-3}$ & $1.4 e^{-3}$ \\
\hline Vega & $1.3 e^{-2}$ & $2.7 e^{-3}$ \\
\hline Delta & $4.3 e^{-2}$ & $1.6 e^{-2}$ \\
\hline Gamma & $8.8 e^{-2}$ & $4.1 e^{-2}$ \\
\hline Rho & $5.3 e^{-3}$ & $2.0 e^{-3}$ \\
\hline Theta & $4.9 e^{-2}$ & $9.2 e^{-3}$ \\
\hline
\end{tabular}

TABLE 3

Differences Between Using Two Rectangular Meshes for Knots

\begin{tabular}{|l|r|r|}
\hline & max rel. diff. & avg. rel. diff. \\
\hline Price & $11 \%$ & $6 \%$ \\
\hline Vega & $15 \%$ & $9 \%$ \\
\hline Delta & $19 \%$ & $11 \%$ \\
\hline Gamma & $27 \%$ & $17 \%$ \\
\hline Rho & $12 \%$ & $7 \%$ \\
\hline Theta & $29 \%$ & $16 \%$ \\
\hline
\end{tabular}

TABLE 4

Relative Difference in Pricing and Hedging Using Constant Volatility

$K \times[0: .33: 2]$. The average price calibrating error for the market call options in this case is .0027. The reconstructed volatility surface using this knot placement is shown in Fig. 7. Comparing Fig. 6 with Fig. 7, the reconstructed volatility surfaces are quite similar in the region $\mathcal{D}$, noting the shape of $\mathcal{D}$. For options with strikes and maturities over the grid $[.85: .1: 1.15] S_{\text {init }} \times[.85: .1: 1.15]$, the relative difference of pricing and hedging factor with the two spline knot placements are shown in Table 3 . We observe that indeed they are acceptably close.

For pricing simple European call/put options, different volatilities are often used in practice to price options of different strikes/maturities in order to accommodate volatility smile. For pricing an exotic option such as a knock-out option, a constant volatility model is inappropriate since the price of this option depends on volatilities of different strikes and maturities. In order to illustrate the potential error in using a constant volatility in pricing exotic options, we simply examine here the price and hedge factors differences between using a constant volatility model and the 1-factor model with the reconstructed volatility function. We use the same S\&P 500 index option example and choose the the arithmetic average (which is 0.1319) of the implied volatilities with $T \leq 2$ as the constant volatility. We compare the prices at a grid $[.85: .1: 1.15] S_{\text {init }} \times[.85: .1: 1.15]$ of strike price and maturity dates (different from given market data). The results are in Table 4 . These two methods give significantly different prices: we notice as much as $11 \%$ relative difference. Similarly all the hedge factors computed using the constant volatility have a large relative difference, we document the results in Table 4. To visualize the difference in detail, we plot the price and hedge factor curves for options with 1-year maturity in Fig.8.

4.3. Incorporate Additional Information. Using market option data to imply the local volatility function in a diffusion model is a look-ahead technique. Frequently, historical data has been used to estimate a constant volatility. The latter is a look-back technique. An interesting question is whether it is possible to combine both techniques to generate better approximation to the true local volatility.

In the proposed spline volatility formulation (7), there are two potential ways that a priori 


\begin{tabular}{|r|r|r|r|r|r|}
\hline Tol & \multicolumn{2}{|c|}{ Regular } & \multicolumn{3}{c|}{ quasi-Newton } \\
\hline & Iterations & Time & Iterations & Time & J evals \\
\hline $1 e^{-2}$ & 7 & 321.29 & 11 & 131.85 & 3 \\
\hline $1 e^{-3}$ & 10 & 401.43 & 18 & 193.91 & 4 \\
\hline $1 e^{-4}$ & 19 & 651.89 & 28 & 286.78 & 5 \\
\hline $1 e^{-5}$ & 25 & 795.45 & 36 & 332.13 & 6 \\
\hline
\end{tabular}

TABLE 5

Quasi Newton results

information can be incorporated. The first is to use the simple bounds to limit the range of the local volatilities at knots. The second possibility is to specify fixed local volatilities at some chosen knots.

We have experimented with setting tighter bounds on the volatility $\bar{\sigma}$ for the S\&P 500 index European call option example. We observe that, as long as the bounds are not too small $(l \leq-.3,, u \geq .3$ in the S\&P 500 example), they can influence volatility values of small $t$ and $s$ far from $S_{\text {init }}$ but do not have much impact in the region $\mathcal{D}$ within which the volatility function is significant in market option prices. However, setting bounds too tight can impede calibrating the market option prices. Therefore, unless one has reliable knowledge on the bounds of the volatilities, they should be sufficiently large to ensure that the calibrating error is sufficiently small. Similar remarks can be made if one wishes to set the volatilities at certain knots to some fixed values.

Finally, we would like to illustrate the potential computational saving by using the quasiNewton updates. In Table 5 we present Matlab computational results for the S\&P index option problem with different tolerances for optimization. We observe significant improvement using quasi Newton approach in computational time. The quasi-Newton approach takes more iterations to converge but requires fewer Jacobian evaluations.

5. Concluding Remarks. Assuming that the underlying asset of options follows a continuous 1-factor diffusion model, we propose a method of accurately approximating the local volatility function $\sigma^{*}(s, t)$ using a finite set of option prices. We emphasize that accurate approximation of the true local volatility surface is crucial in hedging all options (including simple European options) and pricing exotic options. Moreover, since the market option data typically has bid-ask spreads, exact calibration of the option data (the average of the bid-ask spreads) is not necessary and can be harmful.

Based on the formula (6) established in [17, 1], the local volatility function $\sigma^{*}(K, T)^{2}$ is smooth if the European call option value function $v(K, T)$ is sufficiently smooth. We use a spline functional approach to reconstruct the true local volatility function. Choosing the number of spline knots and their placement, we represent a local volatility function $\sigma(s, t)$ by an interpolating spline with a fixed end condition. The volatility values at knots are determined by solving a small nonlinear optimization problem subject to simple bounds. The number of variables in the optimization (7) is not greater than the number of option observations.

We solve the proposed inverse spline approximation optimization problem using a trust region method with the function and Jacobian evaluations using a PDE approach. Computational efficiency through structure exploitation within the framework of finite difference and automatic differentiation is discussed.

We consider two European call options examples illustrating the capability of the proposed method. In the first example, we consider synthetic European call options for which the underlying follows a known absolute diffusion model. Option observation data is simulated by 
evaluating a set of European call options using the analytic formula. The reconstructed local volatility is compared to the true local volatility, indicating a fairly accurate reconstruction in the region within which the local volatility values are significant for option evaluations. With the same example, we illustrate that the constant implied volatility approach can produce incorrect hedge factors even for simple European options. Moreover, when the observable option prices have bid-ask spreads, calibrating market data exactly by using too many spline knots can lead to poor reconstruction of the true local volatility function. In the second example, a S\&P 500 index European call option with market option data of October 1995 is considered. We illustrate the smoothness of the reconstructed local volatility and stability of the proposed method in pricing and hedging.

We have illustrated the potential of the proposed spline volatility approach in discovering the true local volatility function of the underlying from a finite set of option prices. We plan to further investigate automatic techniques for the optimal selection of the number of knots $p \leq m$ and their placement. The importance of the proposed local volatility function reconstruction in pricing exotic options or American options will also be explored.

6. Acknowledgement. We would like to thank Yohan Kim for carefully reading the manuscript and his useful comments. 

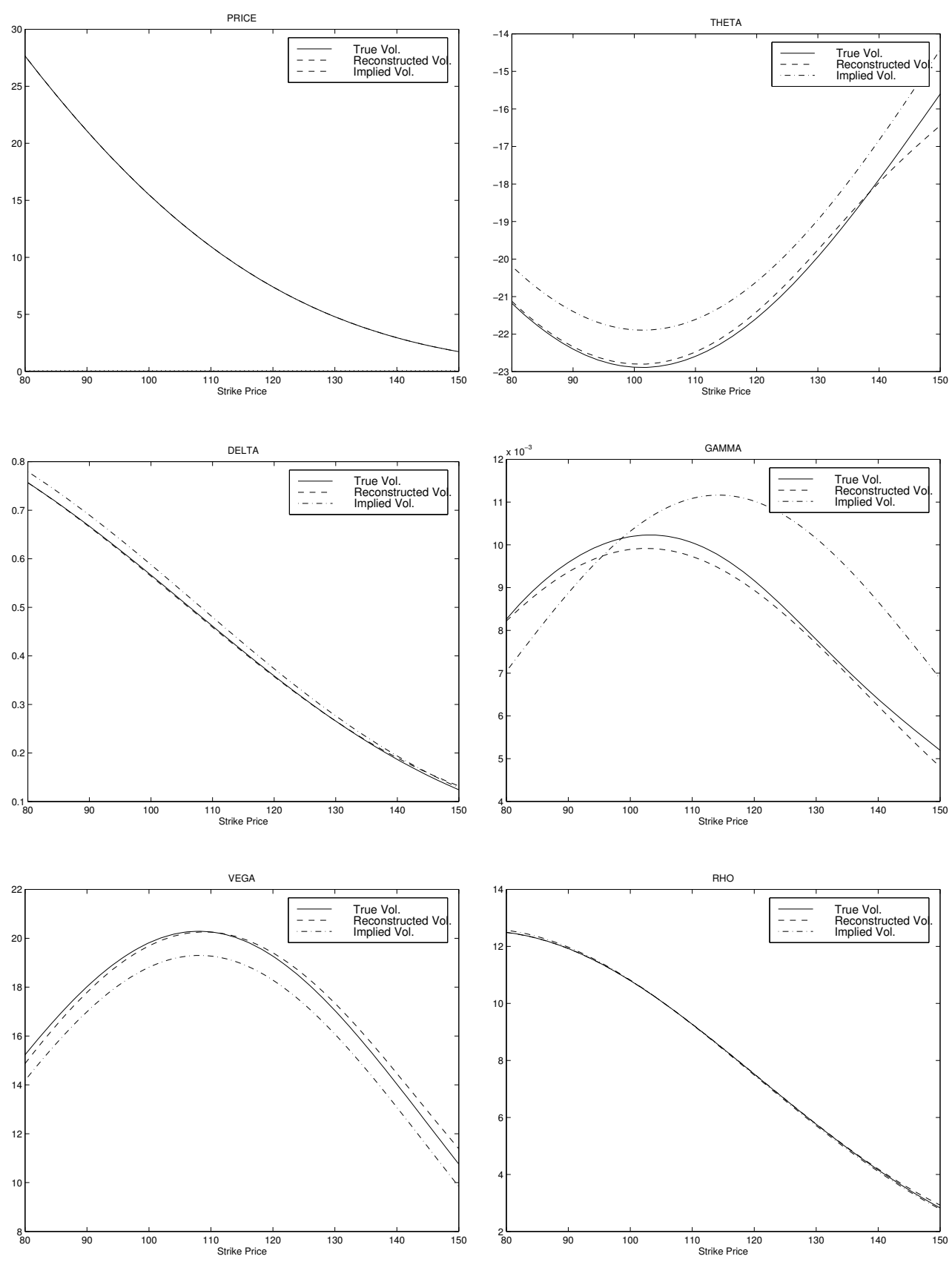

FIG. 5. Comparison Between Using the True, Reconstructed and Constant Implied Volatility Functions 


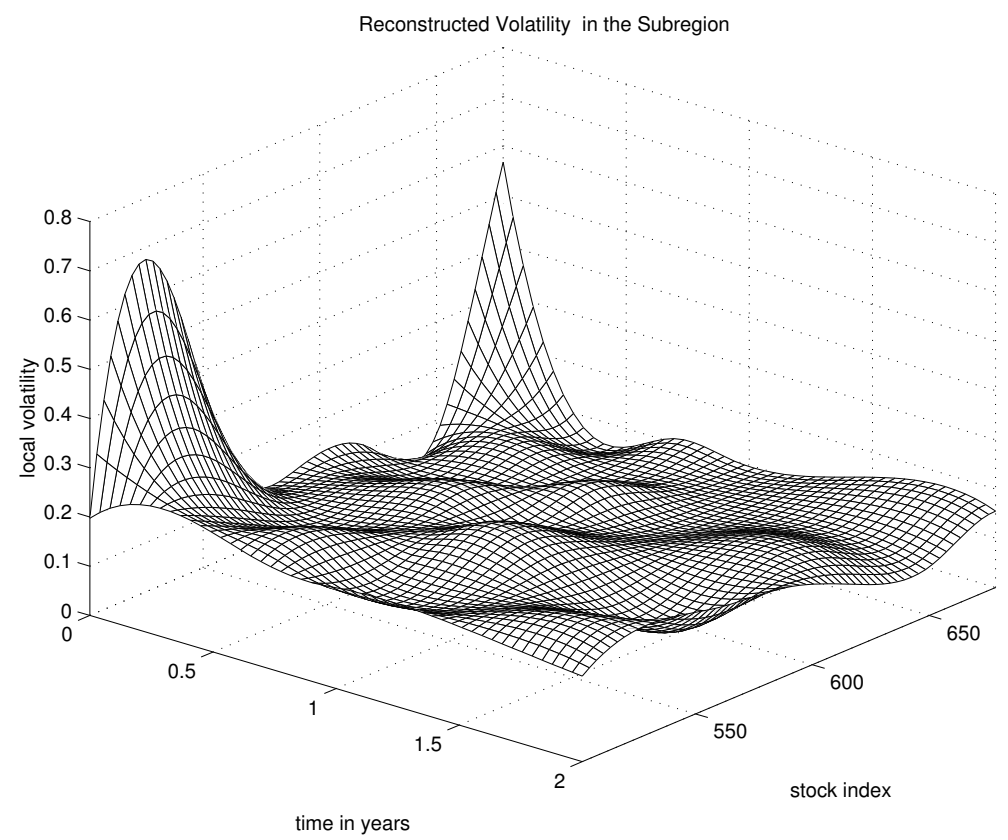

FIG. 6. Spline Knot Placement Is the Rectangular Mesh [.8 $\left.S_{\text {init }}: .066 S_{\text {init }}: 1.4 S_{\text {init }}\right] \times[0: 0.33: 2]$

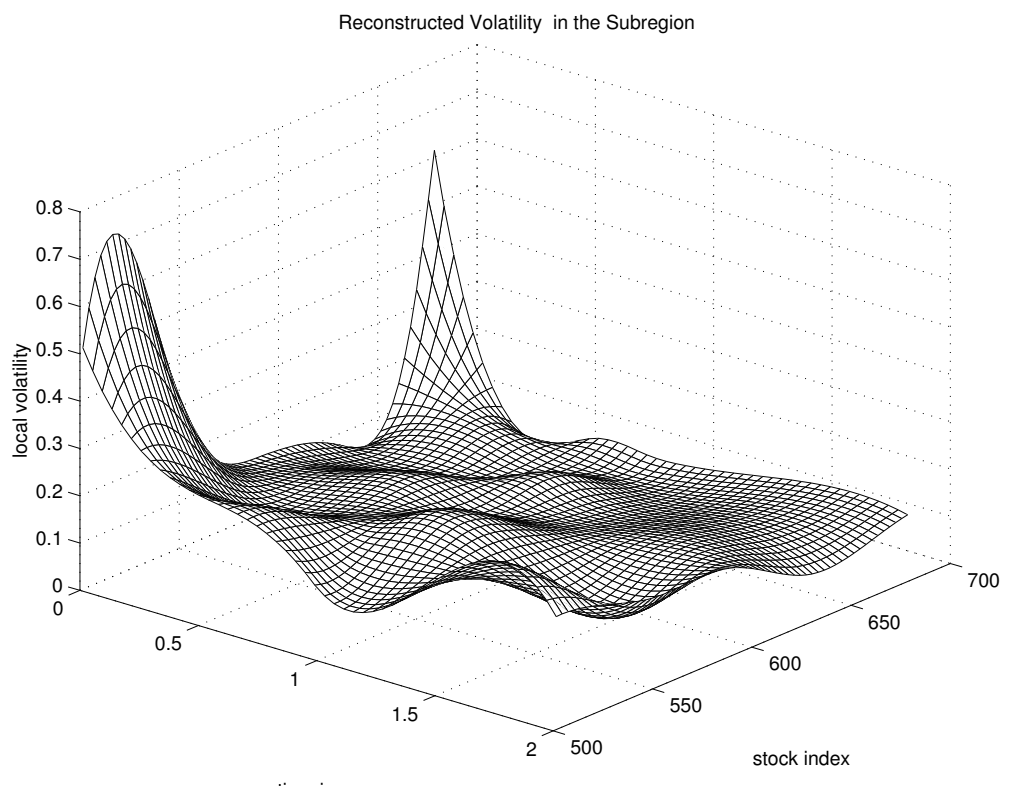

FIG. 7. Spline Knot Placement Is the Rectangular Mesh $K \times[0: 0.33: 2]$ 

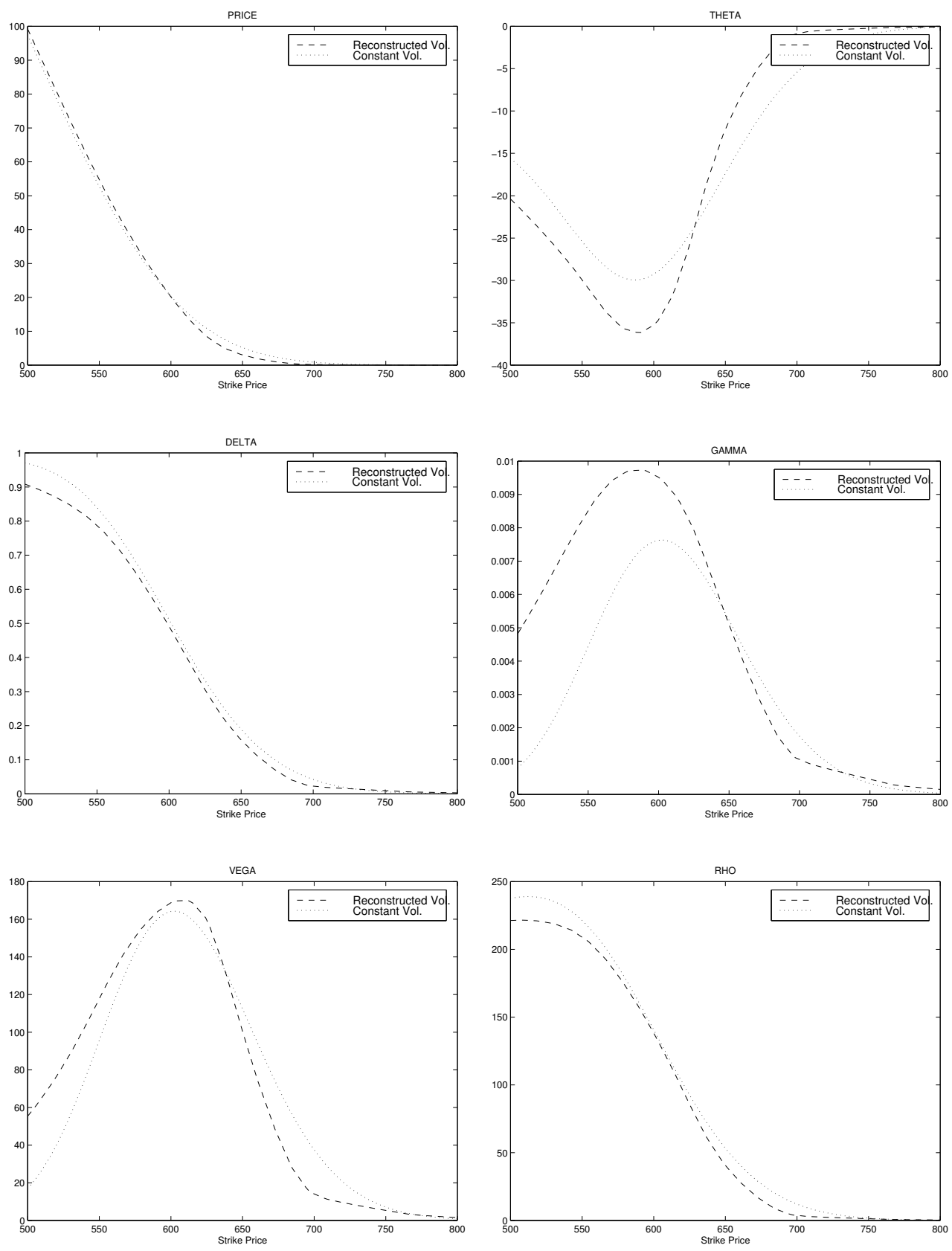

FIG. 8. Comparison Using the Reconstructed Versus Constant (average) Volatility 


\section{REFERENCES}

[1] L. ANDERSEN AND R. BROTHERTON-RATCLIFFe, The equity option volatility smile: an implicit finitedifference approach, The Journal of Computational Finance, 1 (1998), pp. 5-32.

[2] M. Avellaneda, C. Friedman, R. Holemes, And D. SAmperi, Calibrating volatility surfaces via relative entropy minimization, Applied Mathematical Finance, 4 (1997), pp. 37-64.

[3] G. BIRKhoff AND C. R. DE Boor, Piecewise polynomial interpolation and approximation, in Approximation of Functions, H. L. Garabedian, ed., Elsevier (New York), 1965, pp. 164-190.

[4] F. BlaCK AND M. SCHOLES, The pricing of options and corporate liabilities, Journal of Political Economy, 81 (1973), pp. 637-659.

[5] I. Bouchouev And V. Isakov, The inverse problem of option pricing, Inverse Problems, 13 (1997), pp. L11-L17.

[6] M. A. BRAnCh, T. F. Coleman, AND Y. Li, A subspace, interior and conjugate gradient method for large-scale bound-constrained minimization, Tech. Report TR95-1525, Computer Science Department, Cornell University, 1995.

[7] T. F. COlEMAN AND Y. LI, An interior, trust region approach for nonlinear minimization subject to bounds, SIAM Journal on Optimization, 6 (1996), pp. 418-445.

[8] T. F. COLEMAN AND A. Verma, Structure and efficient Jacobian calculation, in Computational Differentiation: Techniques, Applications, and Tools, M. Berz, C. Bischof, G. Corliss, and A. Griewank, eds., SIAM, Philadelphia, Penn., 1996, pp. 149-159.

[9] J. C. CoX AND S. A. Ross, The valuation of options for alternative stochastic processes, Journal of Financial Economics, 3 (1976), pp. 145-166.

[10] S. R. DAS And R. K. Sundaram, Of smiles and smirks: A term-structure perspective, Tech. Report NBER working paper No. 5976, Department of Finace, Harvard Business School, Harvard University, Boston, MA 02163, 1997.

[11] C. DE Boor, Spline Toolbox for use with MATLAB, The Math Works Inc., 1997.

[12] J. E. DENNIS AND R. B. SCHNABEL, Numerical Methods for Unconstrained Optimization, Prentice-Hall, 1983.

[13] E. Derman And I. Kani, Riding on a smile, Risk, 7 (1994), pp. 32-39.

[14] - The local volatility surface: Unlocking the information in index option prices, Financial Analysts Journal, (1996), pp. 25-36.

[15] P. DIERCKX, An algorithm forsurface fitting with spline functions, IMA Journal of Numerical Analysis, 1 (1981), pp. 267-283.

[16] Curve and Surface Fitting with Splines, Oxford Science Publications, 1993.

[17] B. DUPIRE, Pricing with a smile, Risk, 7 (1994), pp. 18-20.

[18] A. GRIEWANK, Some bounds on the complexity of gradients, Jacobians, and Hessians, in Complexity in Nonlinear Optimization, P. Pardalos, ed., World Scientific Publishers, 1993, pp. 128-161.

[19] D. HeAth, R. JARrow, AND A. Morton, Bond pricing and the term structure of interest rates: A new methodology for contingent claims valuation, Econometrica, 60 (1992), pp. 77-105.

[20] J. Hull AND A. White, The pricing of options on assets with stochastic volatilities, Journal of Finance, 3 (1987), pp. 281-300.

[21] J. JACKWERTH AND M. RUBINSTEIN, Recovering probability distributions from option prices, The Journal of Finance, 51 (1996), pp. 1611-1631.

[22] R. Lagnado And S. Osher, Reconciling differences, Risk, 10 (1997), pp. 79-83.

[23] D. LAmberton And B. LApeyre, Introduction to Stochastic Calculus Applied to Finance, Chapman \& Hall, 1996.

[24] R. MERTON, The theory of rational option pricing, Bell Journal of Economics and Management Science, 4 (1973), pp. 141-183.

[25] - Option pricing when underlying stock returns are discontinuous, Journal of Financial Economics, 3 (1976), pp. 124-144.

[26] M. RubinsteIn, Implied binomial trees, The Journal of Finance, 49 (1994), pp. 771-818.

[27] D. Shimko, Bounds of probability, Risk, (1993), pp. 33-37.

[28] A. N. Tikhonov And V. Y. Arsenin, Solutions of Ill-posed Problems, W. H. Winston, Washington, D.C., 1977.

[29] V. N. VAPNIK, Estimation of Dependences Based on Empirical Data, Springer-Verlag, Berlin, 1982.

[30] G. WAнBA, Splines Models for Observational Data, Series in Applied Mathematics, Vol. 56, SIAM, Philadelphia, 1990.

[31] R. ZVAn, K. VETZAL, AND P. Forsyth, Swing high Swing low, Risk Magazine, (1998), pp. 71-74. 